\title{
Correlation Between the Mechanical Behavior of Buried Pipes and Relative Stiffness of the Soil-Pipe System
}

\author{
Pedro Alexandre Guimaraes Rocha ${ }^{1}$, Marcilio Fabiano Goivinho da Silva ${ }^{2}$ \\ ${ }^{1}$ Civil Engineering Department, Federal Institute of Education, Science, and Technology of Sergipe, Brazil \\ ${ }^{2}$ Civil Engineering Department, Federal Institute of Education, Science, and Technology of Sergipe, Brazil
}

\begin{abstract}
To avoid the economic and environmental lost caused by failing buried pipes, it is a must to predict the mechanical behavior of the soil-pipe system. That is because different stiffness between the pipe and its surrounding soil leads to different deformations. The objective of this research is to correlate deformation and failure of buried pipes with the soil-pipe relative stiffness. To achieve a reliable level of precision, a finite element methods analysis was carried out assisted by the software Abaqus. Different physical and mechanical parameters of both pipe and soil were tested on the FEM software in order to find the desired correlation. When discretizing the soil, an adaptive mesh was necessary. Thus, regions with differently sized elements were created where discontinuity was found to be high. By computing the results of this study, two points were observed. First, ratios of relative stiffness lesser than 10 have a failure controlled by the pipe since external forces are resisted by this structure. Second, a correlation between deformation/failure and relative stiffness of the system was established. Therefore, this research presents a simple and reliable approach in analyzing the failure mode of buried pipes.
\end{abstract}

Keywords: Buried pipes, finite element methods, relative stiffness.

\section{Introduction}

Buried pipes are commonly used to transport derivatives of petroleum, natural gas and water. Its failure may cause economic and environmental losses since the detection procedure of rupture is imprecise and maintenance works are expensive. In order to avoid disruption of buried pipes, the purpose of this research resides on relating the relative stiffness of the soil-pipe system with the different possible failure modes. As soil is a non-homogenous material, that correlation brings a simple and reliable method to predict the response of buried pipes. The usage of a finite element methods software relies on its accurate results that leads to rigorous analyzes. For instance, by using the software Abaqus, the soil-pipe interaction can be modeled and critical points can be identified. As a result, if a correlation is found, the process of designing a buried pipe will be optimized.

\section{Soil-Pipe System}

The difference in rigidity of the soil-pipe system leads to a redistribution of stress near the pipe and causes different layouts of deformation. Thus, it's necessary to investigate the soil-pipe system to predict the final response of a buried pipe. As an approach for this paper, the relative stiffness of the system will be correlated to rupture and deformation. Accordingly to Allgood and Takahashi (1972), the relative stiffness $(R R)$ can be classified based on the stiffness of the transverse section of the pipe $\left(R_{c}\right)$, and the stiffness of the surrounding soil $\left(R_{s}\right)$. Equations for $R R(1), R_{c}(2)$ and $R_{s}(3)$ are presented below.

$$
\begin{aligned}
& R R=\frac{R_{g}}{R_{e}} \\
& R_{c}=\frac{E_{p} I}{D^{g}} \\
& R_{s}=\frac{E_{s}}{\left(1-v_{s}\right)}
\end{aligned}
$$

Where: $E_{p}$ is the Young modulus of the pipe material, $I$ is the moment of inertia of the pipe wall, $D$ is the diameter of the pipe, $E_{s}$ is the soil modulus of elasticity, and $v_{s}$ is the Poisson coefficient of the surrounding soil. By varying pipe dimensions - diameter and wall thickness - and soil parameters, different stiffness for the soil-pipe system were achieved. Properties such as permeability, interface friction coefficient, density, and undrained shear strength were found to play an important role when characterizing the soil and the failure mode. As to minimize computational time, limitations on the physical dimensions of both soil and pipe were required. For instance, one layer of soil was considered per analysis, and physical and mechanical parameters of the pipe were chosen based on commercial availability. As a result, different soils and pipes were modeled to find a correlation between relative stiffness of the system and failure. 


\section{The Finite Elemnt Methods Model}

Soil is a nonhomogeneous material, so its mechanical behavior cannot be considered linear. Furthermore, because of the complexity of soils, a parameter can be applicable for a certain problem but limited to others. Hence, to develop a representative model, material parameters, structure mesh, loading steps and interaction properties must be cautiously designed.

When modeling the plastic behavior of the soil-pipe system, the Mohr-Coulomb yield criterion was chosen because of its simplicity and accuracy. Besides the yield criterion, parameters such as permeability, elasticity, density, and friction angles are crucial to determine the mechanical behavior of soils. Thus, they were gathered from Bowles (1996), see Table 1 and 2.

Table 1. Young modulus of a soil layer adapted from Bowels (1996)

\begin{tabular}{|c|c|c|}
\hline Soil Type & Characteristics & $\mathrm{E}_{\mathrm{s}}(\mathrm{MPa})$ \\
\hline \multirow{3}{*}{ Clay } & Very soft & $2-15$ \\
\cline { 2 - 3 } & Soft & $5-25$ \\
\cline { 2 - 3 } & Medium & $15-50$ \\
\cline { 2 - 3 } & Stiff & $50-100$ \\
\hline \multirow{4}{*}{ Sand } & Loose & $10-25$ \\
\cline { 2 - 3 } & Dense & $50-81$ \\
\hline
\end{tabular}

Table 2. Poisson ratio adapted from Bowels (1996)

\begin{tabular}{|c|c|c|}
\hline Soil Type & Characteristics & $v_{s}$ \\
\hline \multirow{2}{*}{ Clay } & Saturated & $0.4-0.5$ \\
\cline { 2 - 3 } & Unsaturated & $0.1-0.3$ \\
\hline \multirow{2}{*}{ Sand } & Loose & $0.2-0.35$ \\
\cline { 2 - 3 } & Dense & $0.3-0.4$ \\
\hline
\end{tabular}

When defining the steps, two of them were created. The initial step was modeled with predefined void ratios, and the geostatic one to account for consolidation and/or settlements of the soil layer. In the mesh, tetrahedral elements (C3D10) were set for both pipe and soil since they present reasonable precision when deformed. The mesh was created in partitions with differently sized elements (Fig. 1 and 2). Where discontinuity and distortions were found, the mesh was refined. In addition, the elements were set to allow pore pressure and fluid stress as part of their degree of freedom; i.e., soil consolidations were analyzed.

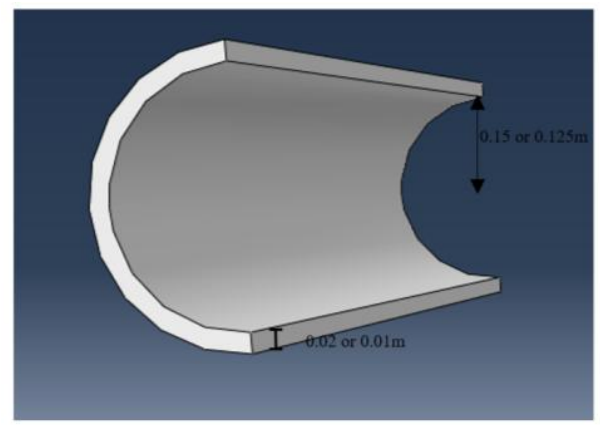

Figure 1. Detail of the modeling of the buried pipe

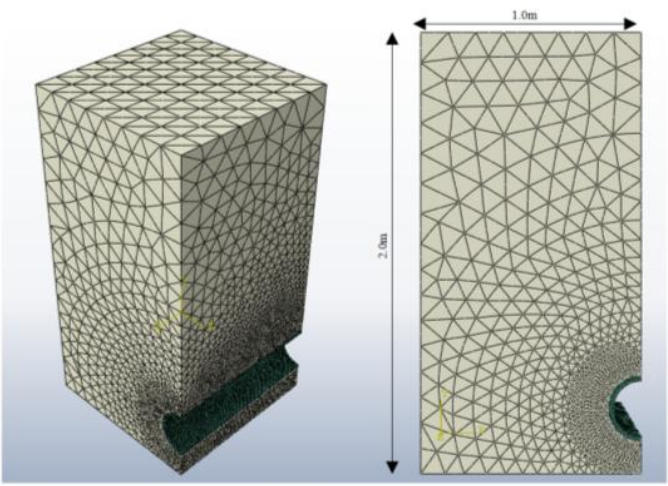

Figure 2. Meshed structure in perspective and plan view 
For the soil-pipe contact properties, normal and tangential behaviors were set with the penalty constraint enforcement method. The normal behavior was decided as a "hard" contact. Furthermore, friction coefficients between the soil and pipe surface were set based on the type of soil and taken from Hikooei (2013). Finally, 76 different types of soil-pipe systems were modeled in which mechanical properties varied from model to model, see Table 3 .

Table 3. Quantity of tested models per category of relative stiffness

\begin{tabular}{|c|c|}
\hline Relative Stiffness & Quantity of different layouts modeled \\
\hline RR $<10$ & 23 \\
\hline $10<\mathrm{RR}<100$ & 39 \\
\hline RR $>100$ & 14 \\
\hline Total & 76 \\
\hline
\end{tabular}

\section{Validation Of The Fem Model}

Analytical methods were used and compared to the FEM model in order to create a representative analysis. The German method (Jeyapalan and Hanida, 1988) and the Watkins method (Watkins and Anderson, 1999) were vital to calculations. For the German method (Eq. 4), the vertical stress applied on the buried pipe can be approximated from the specific weight of the upper soil $(\gamma)$, the width $\left(B_{v}\right)$ and the load factor $(C v)$ of the ditch, and an $L$ factor that depends on the relative stiffness of the soil-pipe system.

$\sigma_{v}=\gamma B_{v} L C_{v}$

For the Watkins method (Eq. 5), deflections of buried pipes can be predicted from the relative stiffness of the system $(R R)$, and factors a and $\mathrm{b}$ that accounts for the surrounding soil $\left(F_{d}\right)$ and the creep factor of the pipe material $\left(F_{k}\right)$.

$\frac{a_{y}}{\varepsilon_{y}}=\frac{R R}{a+b \times R R}$

Parameters such as pressure and deformation of the pipe were calculated from analytical methods and were compared to the results obtained from the finite element analysis. As they were found to be similar, the accuracy of the model was corroborated.

\section{V. $\quad$ Failure And Relative Stiffness}

On Figure 3, it is possible to verify the distribution of stress after the placement of a buried pipe throughout the finite elements method software.
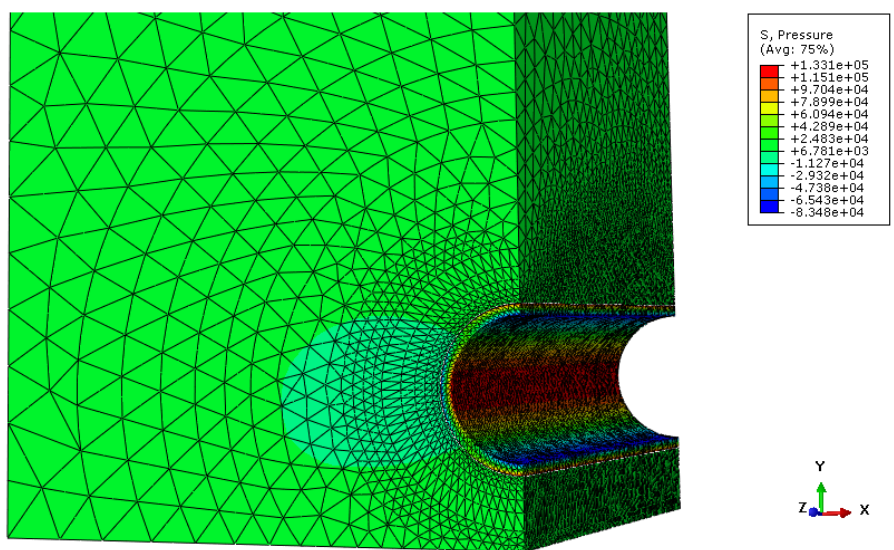

Figure 3. Distribution of stress on the soil-pipe system

It was possible to compare the deformation from the FEM software with the one from calculations. Furthermore, it was evident from the comparison that the model is representative of what can be found on the literature. On Figure 4, it is presented the layout of deformation of the soil pipe system on the $\mathrm{x}$-axis. 

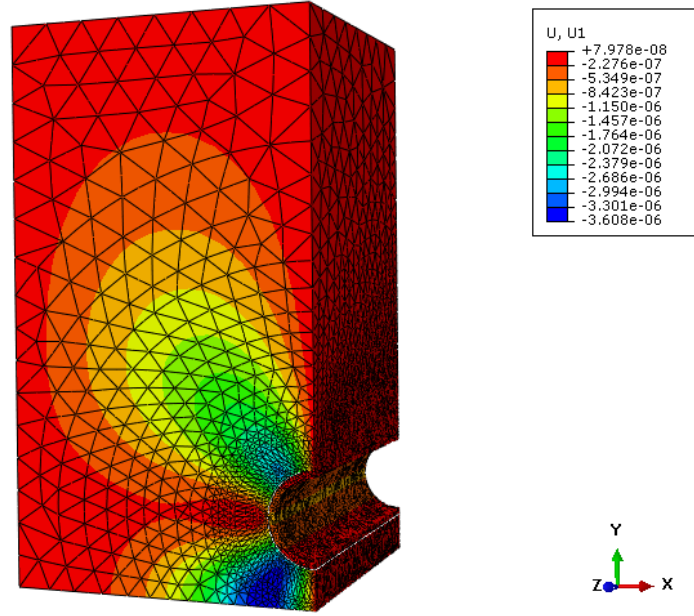

Figure 4. Deformation of the soil-pipe system on the x-axis

Allgood and Takahashi (1972) stated how to define the relative stiffness of the soil-pipe system. Afterwards, Gumbel et al. (1982) found a relationship between relative stiffness and the soil-pipe system behavior. That correlation is presented on Table 4 .

Table 4. Relative stiffness and the mechanical behavior of the system

\begin{tabular}{|c|c|c|}
\hline Relative Stiffness & Supported load by the pipe & Behavior of the system \\
\hline RR $<10$ & More than $90 \%$ & Rigid \\
\hline $10<\mathrm{RR}<1000$ & From $10 \%$ to $90 \%$ & Intermediary \\
\hline RR $>1000$ & Less than $10 \%$ & Flexible \\
\hline
\end{tabular}

The possible types of failure of buried pipes are due to local buckling, crushing, excessive deflection, and cracking of the pipe wall (Rubio, 2008). In Figure 5, it's illustrated the proposed relation between failure mode of the structure based on the rigidity of the soil and the pipe.

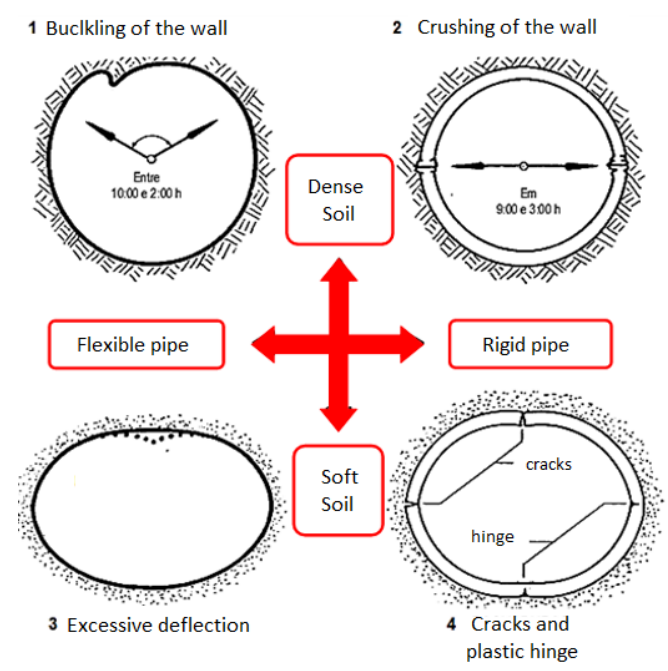

Figure 5. Failure modes and the external pressure from the soil (Adapted from Rubio, 2008)

From the finite element analysis of different structures, the qualitative correlation within the relative stiffness of the soil-pipe system and its behavior was found, see table 5 .

Table 5. Correlation between relative stiffness and behavior of the soil-pipe system

\begin{tabular}{|c|c|}
\hline Relative Stiffness & Behavior of the system \\
\hline RR $<10$ & Crushing of the pipe wall \\
\hline $10<$ RR $<100$ & Local buckling of the pipe \\
\hline RR $>100$ & Excessive deflection of the pipe \\
\hline
\end{tabular}


As a result, the response from the finite element methods analysis is in accordance with both Rubio (2008) and Gumbel et al. (1982). For example, when the relative stiffness was lesser than 10 in the fem analysis, the soil-pipe system was rigid and controlled by crushing in the pipe.

\section{Conclusion}

The damages caused by the failure of a buried pipe can generate losses beyond the economic level. As soil is a nonhomogeneous material, its comportment is uncertain after redistribution of stress instigated by a buried pipe. This research has proven that the finite element software is a reliable instrument when analyzing the mechanical behavior of soil-pipe systems. Furthermore, a qualitative correlation between rupture/deformation and relative stiffness of the structure has been created. As a result, by knowing few parameters of both soil and pipe, the failure mode may be consistently predicted.

\section{Future Research}

On this research, a qualitative correlation has been made between the failure mode of a buried pipe and the relative stiffness of the soil-pipe system. Proceeding works are being carried out in order to set up a correlation between the pipe deformation and the relative stiffness of the system.

\section{Acknowledgements}

First, I would like to express my gratitude to the Federal Institute of Sergipe for technical and financial supports. Furthermore, I would like to express my gratitude to my professor Marcilio Goivinho for his guidance and valuable advices. Lastly but not least, I would like to thank my family and friends for their endless encouragement.

\section{References}

[1] Allgoog, R. J.; Takahashi, S. K. Balanced design and finite element analysis of culverts. Highway Research Record, HRB, n. 413, p. $45-56,1972$

[2] Bowles, J. E., Foundation analysis and design. 5. Ed: McGraw-Hill, 1996. New York, USA.

[3] Chevalier, B.; Combe, G.; Villard, P., 2009. Experimental and numerical studies of load transfers and arching effect in the trapdoor problem. Laboratoire Sols, Solides, Structures - Risques, Grenoble, France.

[4] Conrad, B. E.; Lohnes, R. A.; Klaiber, F. W.; Wipf, T. J., 1998. Boundary effects on response of polyethylene pipe under simulated live load. Transportation Research Record 1624, p. 196-205.

[5] Costa, Y. D. J., 2005. Modelagem física de condutos enterrados sujeitos a perda de apoio ou elevação localizada. Tese de D.Sc. em Geotecnia, Escola de Engenharia de São Carlos, São Carlos, Brasil.

[6] Getzler, Z.; Korormik, A.; Mazurik, A., 1968. Model study in arching above buried structures. Journal of the Soil Mechanics and Foundation Division. n. SM5. p. 1123-1141.

[7] Gumbel, J. E.; O’Reilly, M. P.; Lake, L. M.; Carder, D. R. The development of a new design method for buried flexible pipes. Proceedings of the Europipe'82, p. 87-98, 1982.

[8] Hikooei, B. F., 2013. Numerical modeling of pipe-soil interaction under transverse direction. Master's thesis, University of Calgary, Calgary - Alberta, Canada.

[9] Hill, R., 1998. The mathematical Theory of Plasticity. Oxford Classics Texts in the Physical Sciences, Nova York, EUA.

[10] Hobbit, Karlsson \& Sorensen, 2003a. Abaqus 6.8 - Theory Manual, Inc. Pawtucket, USA.

[11] Hobbit, Karlsson \& Sorensen, 2003b. Abaqus 6.8 - User's Manuals I, II e III, Inc. Pawtucket, USA.

[12] Jeyapalan, J. K.; Hamida, H. B. Comparison of German to Martson design methods. Journal of Transportation Engineering, v. 114, n. 4, p. 420-434, 1988.

[13] Marston, A., 1930. The theory o external loads on closed conduits in the light of the latest experiments. Iowa Engineering Experimental Station, Ames, Bulletin 96, 36 p., EUA.

[14] Mckelvey III, J. A., 1994. The anatomy of soil arching. Geotextiles and Geomembranes, v.13, p.317-329.

[15] Nielson, F. D., 1967. Soil Structure Arching Analysis of Buried Flexible Structures. Highway Research Board, HRB 185, p.36-50.

[16] Ono, K.; Yamada, M., 1993. Analysis of the arching action in granular mass. Geotèchnique, 43 n1 105-120.

[17] Rogers, C. D. F., 1988. Some observations on flexible pipe response to load. Transportation Research Record 1191, p. 1-11.

[18] Rubio, N. P., 2008. Estudo de dutos enterrados considerando a interação solo-estrutura. PhD thesis, PUC-Rio, Rio de Janeiro - RJ, Brazil.

[19] Santichaianaint, K., 2002. Centrifuge modeling and analysis of active trapdoor in sand. Tese de Ph.D., Department of Civil, Environmental and Architectural Engineering, University of Colorado at Boulder, EUA.

[20] Sloan, S, W.; Assadi, A.; Purushothaman, N., 1990. Undrained stability of a tropdoor. Géotechnique, v. 40, n. 1, p.45-62.

[21] Strömblad, N., 2014. Modeling of soil and structure interaction subsea. Master's thesis in applied mechanics, Chalmers University of Technology, Göteborg, Sweden.

[22] Terzaghi, K., 1943. Theoretical soil mechanics. John Wiley \& Sons, New York, EUA.

[23] Watkins, R. K., 1975. Buried Structures-Foundation Engineering. Handbook, p.649-672.

[24] Watkins, R. K.; Anderson, L. R. Structural mechanics of buried pipes. 1. Ed. [S.1.]: GRG Press, 1999. 